\title{
Trust in organization effect on the relationship between HRM practices and employee well-being
}

\author{
Vaida Jaškevičiūtè $\dot{1}^{*}$ \\ ${ }^{1}$ The Department of Management, Faculty of Economics and Business Administration, Vilnius \\ University, Saulètekio av. 9, 2nd building, 10222, Vilnius, Lithuania.
}

\begin{abstract}
This study investigates the effects of trust in the organization on the relationship between human resource management practices and employee well-being. Method: An anonymous survey of 638 employees in Lithuania was conducted. The research was designed based on a 6dimensional scale of perceived human resources management policies and practices, a 3-dimensional questionnaire of employee well-being, and a 2dimensional questionnaire of trust in the organization. Questionnaire items were measured on a 5-point Likert scale. Survey data were analyzed using descriptive statistics, correlations, multivariate linear regressions and mediation analysis. Findings: Specific relationships were found between various HRM practices with employee well-being in having a full effect or in having a partial effect. This empirical study showed that select HRM practices positively influence employee well-being through the mediating effect of trust in the organization. Managerial implications: Based on the results of the survey, employee well-being at work in Lithuania can best be improved by the strengthening of trust in the organization through following these HRM practices: "recruitment and selection" (RS), "involvement" (I), "work conditions" (WC) and "competence-based performance appraisal" (CBPA). Keywords: human resource management, impersonal trust, wellbeing of employees, Lithuania.
\end{abstract}

\section{Introduction}

Special attention has been paid to various topics of human resource management (HRM) in the scientific literature for over twenty years. Employees are considered an essential factor of the organization's success [1]. The preservation of employees in the organization by ensuring their well-being should be one of the primary goals of the organization [2]. The employee well-being (EWB) has been found to relate positively to various individual and organizational outcomes [3-5]. One of the most effective ways to achieve EWB is through the application of HRM practices that are oriented towards the employee's well-being in the activity of the organization [1]. The importance of trust is also accentuated in scientific literature, and employee trust is related positively with HRM practices and with EWB [6-9]. Most research assesses interpersonal trust without assessing the influence of impersonal trust. For this reason, the aim of this article is to uncover the impact of trust in the organization on

\footnotetext{
* Corresponding author: vaida.jaskeviciute@evaf.vu.lt
} 
the relationship between different HRM practices and employee well-being in Lithuanian organizations.

\section{Theoretical background}

\subsection{Human resource management practices}

The relationship, based on social exchange theory, between HRM practices and EWB is equivocal. Several studies have found a positive relationship between HRM practices and EWB [10-12]. Based on Agarwal and Farndale [13], "HRM practices increase psychological resources in the form of self-efficacy, optimism, hope, resilience, and psychological safety, which in turn are likely to increase EWB. Luthans [13] argued that HRM practices enhance EWB by mobilizing employees' motivational and cognitive resources" [13, p.441-444]. Other studies in social and business fields have suggested that the effects of HRM practices on EWB depend on the configuration of the HRM system [14-15, 3]. For example, HPWS or HCWS are negatively related to EWB because they promote employees' perceived work load, level of stress, pressure to meet goals, work-family conflict, etc. [5, 16, 4]. HRM practices also depend on contextual factors (e.g., nature of organization's activity, size of organization, etc.) [4]. Some HRM practices dominate more, others less. For this reason, it is important to explore in greater detail the effects of various individual practices.

The HRM practices emphasized by Demo et al. [17] are analyzed in the context of this study: "recruitment and selection" (RS), "involvement" (I), "training, development \& education" (TD\&E), "competence-based performance appraisal" (CBPA) and "compensation and rewards" (CR) [17]. These practices were not chosen accidentally. The main reason these practices were chosen is that these HRM practices are oriented towards the results of the organization by taking into account the well-being of employees. These HRM practices reflect the essence of the "mutual gain model" - a mutual benefit for the organization and its employees is achieved [1, 18]. The organization's managers have a proactive employment role in encouraging organizations to develop HRM practices focused on EWB, as though these types of actions they increase employee productivity and reduce the desire to leave organizations, additionally, improved employee health.

\subsection{Employee well-being}

In the scientific literature it can be found that the employee well-being is superposed with job satisfaction [19-20]. Job satisfaction is defined as a positive emotional state arising from the assessment of employee work or work experiences [21]. Fisher [22] relate job satisfaction with the satisfaction-causing aspects of work that lead to better work productivity. Taking into account the definition of job satisfaction, it can be stated that job satisfaction is something like an indicator that enables the representatives of the organization to find out if the employee is satisfied with the performed activity and able to realize himself/herself. Job satisfaction is also important to employees because it reflects on the relationship to the employees' expectations towards work. In the opinion of most scientists, it is too narrow to compare an employee's job satisfaction with an employee's well-being [23-24, 12, 20]. According to Page and Vella-Brodrick [23], Zheng at al. [24], job satisfaction is just one constituent of workplace well-being. Thus, workplace well-being is one of the dimensions of the comprehensive employee well-being construct [23-24].

Scientists do not have a unanimous opinion on what exactly employee well-being is. The conception of employee well-being is related with a multi-component construct involving complex interactions of various factors. Thus, the phenomenon of employee well-being is 
defined through its constituents. In the opinion of scientists, the construct that reflects the comprehensive essence of employee well-being consists of life well-being (such as subjective well-being), workplace well-being and psychological well-being [23-24, 12]. This employee well-being construct can be characterized with a holistic attitude that accentuates the employee well-being will be ensured and assessed properly in the case when all the individual constituents function well together. The synergy created by the dimensions of the employees' well-being construct enables the employees of the organization to feel full-fledged, emotionally healthy, non-conflicting among different obligations, and available for selfrealization.

\subsection{Trust in organization}

The conception of trust is understood as a personal intention to accept one's own vulnerability in relation to the acts of another party that is trusted. This attitude is based on the expectations and belief that these acts will be useful, favorable, or at least un-harmful to the other party [25-26]. In the context of the organization, trust is classified as interpersonal trust and impersonal trust. Interpersonal trust consists of the trust in co-workers and trust in managers, while impersonal trust - trust in the organization [27-30]. In the scientific literature, the trust in managers is mostly accentuated [31-32], while the trust in co-workers is accentuated somewhat less [33]; trust in the organization is a new phenomenon that needs more comprehensive empirical research [28]. In this article, attention is paid to impersonal trust.

According to Paliszkewicz [34], trust in the organization is the employee's belief that the other party: 1) will not do anything that would be harmful to the trusting party; 2) will behave in a way that would be useful to the trusting party; 3 ) will behave with trustworthiness; 4) will behave or react in a predictable way that would be acceptable to both parties [34]. Taking this into account, it can be stated that trust in the organization is that the relations between an employee and the organization will be benevolent and mutually useful [34-35].

Trust in the organization is especially important in the modern world where employees become more and more mobile, i.e., employees can work in different places and at any time of the day. For this reason, interpersonal trust decreases and the importance of impersonal trust increases. According to Chen et al. [36], trust in the organization is an informal agreement between an employee and the organization. Employees that trust in the organization feel safer, their productivity is higher [37] and it enables employees to give a sense of value to their work [35]. Trust in the organization is equated with healthy organization-employee relationships that are associated with the well-being of employees [9]. There is still a lack of research and empirical substantiation on the effect on trust in the organization in order to achieve EWB. Taking this into account, the effect of trust in the organization as a mediating variable, investigating the correlations between the different HRM practices and employee well-being, was explored in this study. Accordingly, the author's hypothesis:

Hla-f: Trust in the organization effect on relationship between HRM practices: "RS" (la), "I" (1b), “TD\&E” (1c), “WC" (1d), "CBPA" (1e), "CR" (1f) and employee well-being.

Figure 1 demonstrates the hypothesized relationships. 


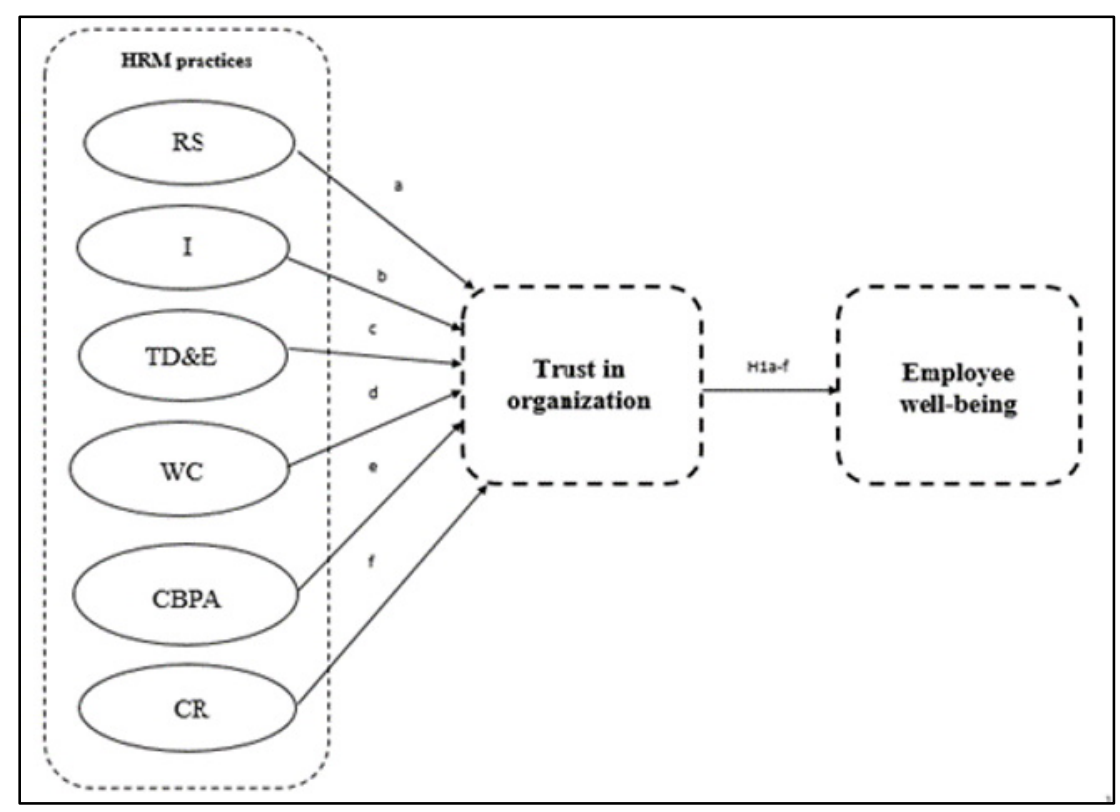

Fig. 1. Summary of hypothesized relationships (source: compiled by author).

Note: HRM practices: RS "recruitment and selection", I "involvement", TD\&E "training, development \& education", WC "work conditions", CBPA "competence-based performance appraisal", CR "compensation and rewards".

\section{Methodology}

\subsection{Source of data}

A quantitative survey was used to conduct this study. The online questionnaire was distributed via an online survey portal www.apklausa.lt. Respondents were asked to fill in the questionnaire only if they were working in organizations of Lithuania. In total, 638 questionnaires were collected for this study. Survey data was collected in January-February 2021.

\subsection{Measures}

The questionnaire consisted of four thematic blocks: HRM practices, employee well-being, trust in the organization and demographics. The demographic section included multiplechoice questions identifying gender, age, education, size of organization and the type of business activity. All of the study constructs and measurements were based on the existing literature. The questionnaire items were designed as statements, measured in a 5-point Likert scale, from 1 "strongly disagree" to 5 "strongly agree".

Employee well-being was measured with an 18 -item scale $(\alpha=0,88)$, developed by Zheng et al. (2015) [24]. This scale consists of three dimensions: life well-being (6-items), workplace well-being (6-items) and psychological well-being (6-items).

HRM practices were measured with a 40 -item scale, developed by Demo et al. [17]. HRM practices were divided into 6 dimensions, according to Demo et al. [17]: 1) "RS" (6items; $\alpha=0,83)$; 2) "I" (12-items; $\alpha=0,89) ; 3$ ) "TD\&E" (6-items; $\alpha=0,84) ; 4)$ "WC" (6-items; $\alpha=0,86)$; 5) "CBPA" (5-items; $\alpha=0,85) ; 6$ ) "CR" (5-items; $\alpha=0,85$ ). 
Trust in organization was measured with 31 -items scale $(\alpha=0,94)$, developed by Vanhala et al. [28]. This scale consists of two dimensions of the impersonal form of trust: capability (18-items) and fairness (13-items).

All of the study's construct validity were based on existing literature $[17,24,38]$ and do not require validation.

\subsection{Data analysis}

Quantitative survey data were processed using a statistical data analysis program IBM SPSS Statistics 25. Survey data were analyzed using descriptive statistics, correlations, multiple linear regressions, and mediation analysis. To assess the mediation formally, the author applied the bootstrapping procedure, using the PROCESS Procedure for SPSS Version 3.5.2 Model 4 by Andrew F. Hayes, to estimate the confidence interval of the indirect relationship.

\section{Findings}

\subsection{Sample profile}

The survey included 638 respondents, of which $60,5 \%$ were female and 39,5\% male. More than one third of respondents were aged between $41-50$ years old $(34,3 \%)$. The second largest group was aged between 31-40 years $(28,5 \%)$, following next were the age groups 51-60 $(18,5 \%), 18-30(14,1 \%)$, and finally the smallest group was $60-64(4,7 \%)$. The largest proportion of respondents $(82,8 \%)$ had a university education, others $(17,2 \%)$ had a collage or lower education. Based on the size of organization, half of the respondents were working in middle-size organizations (50,2\%) of 50-249 employees, $23,4 \%$ worked in large organizations of greater than 250 employees. The remaining respondents worked in small (10-49 employees) organizations, $21,5 \%$, or micro-organizations (less than 10 employees), $5,0 \%$. The business activity distribution was as follows: service $(36,1 \%)$, manufacturing $(29,3 \%)$, trade $(19,9 \%)$ and finally, mixed activity $(14,7 \%)$.

\subsection{Descriptive statistics}

The HRM practice "TD\&E" $(M=3,55 ; \mathrm{SD}=0,716)$ was evaluated as the highest among all six HRM practices. The second was the HRM practice "I" $(\mathrm{M}=3,44 ; \mathrm{SD}=0,709)$. The lowest mean was HRM practice "CR" $(\mathrm{M}=2,80 ; \mathrm{SD}=0,817)$. Other HRM practices means were: "CBPA" (M=3,34; $\mathrm{SD}=0,782)$; "RS" (M=3,37; $\mathrm{SD}=0,670)$ and "WC" $(\mathrm{M}=3,16 ; \mathrm{SD}=0,823)$. The mean value of employee well-being was rather high $(\mathrm{M}=3,60 ; \mathrm{SD}=0,576)$. Respondents report their trust in the organization was also indicated as rather high $(\mathrm{M}=3,38 ; \mathrm{SD}=0,596)$.

\subsection{Correlation analysis}

The correlations between HRM practices, EWB and trust in the organization, were explored. Table 1 presents correlation analysis.

Table 1. Correlation analysis (source: compiled by author from survey data) 


\begin{tabular}{|l|l|l|l|l|l|l|l|l|}
\hline & RS & I & TD\&E & WC & CBPA & CR & EWB & Trust \\
\hline RS & 1 & & & & & & & \\
\hline I & $0,590^{* *}$ & 1 & & & & & & \\
\hline TD\&E & $0,560^{* *}$ & $0,649^{* *}$ & 1 & & & & & \\
\hline WC & $0,453^{* *}$ & $0,632^{* *}$ & $0,523^{* *}$ & 1 & & & & \\
\hline CBPA & $0,629^{* *}$ & $0,586^{* *}$ & $0,595^{* *}$ & $0,479^{* *}$ & 1 & & & \\
\hline CR & $0,392^{* *}$ & $0,562^{* *}$ & $0,431^{* *}$ & $0,430^{* *}$ & $0,508^{* *}$ & 1 & & \\
\hline EWB & $0,217^{* *}$ & $0,450^{* *}$ & $0,297^{* *}$ & $0,357^{* *}$ & $0,335^{* *}$ & $0,332^{* *}$ & 1 & \\
\hline Trust & $0,431^{* *}$ & $0,710^{* *}$ & $0,532^{* *}$ & $0,528^{* *}$ & $0,525^{* *}$ & $0,633^{* *}$ & $0,537^{* *}$ & 1 \\
\hline
\end{tabular}

${ }^{* *}$ Correlations were statistically significant $(p<0,01), N=638$. Trust-trust in the organization.

The results showed that HRM practices: "RS", "I", "TD\&E", "WC", "CBPA", "CR", EWB, and trust in the organization had medium or weakly positive, and statistically significant correlations with each other.

\subsection{Regression analysis}

To examine whether HRM practices are statistically significant associated with EWB, the author conducted multiple regression analyses with EWB as the dependent variable. Some predictors show substantial intercorrelations. The author checked for possible biases due to multicollinearity. Variance inflation factors (VIFs) fell far below the critical value of 10 [39] for all regression models (VIF $\leq 4)$. Further multiple regression analyses demonstrate that not all variables are statistically significantly interrelated. Table 2 presents factors, coefficients, and significance for the final regression model.

Table 2. Coefficients of the final regression models (source: compiled by author from survey data)

\begin{tabular}{|c|c|c|c|c|c|c|}
\hline \multicolumn{7}{|c|}{ Model summery } \\
\hline \multirow{2}{*}{\multicolumn{2}{|c|}{$\mathbf{R}^{2}$}} & \multicolumn{2}{|c|}{ ANOVA } & & & \\
\hline & & F & $p<0,05$ & & & \\
\hline 0,230 & & 47,169 & 0,00 & & & \\
\hline \multicolumn{7}{|c|}{ Cofficients of the regression model } \\
\hline & & $\begin{array}{l}\text { Unstanda } \\
\text { coefficie: }\end{array}$ & $\begin{array}{l}\text { lized } \\
\text { s }\end{array}$ & $\begin{array}{l}\text { Standardized } \\
\text { coefficients beta }\end{array}$ & $\mathrm{t}$ & $\mathrm{p}=0,05$ \\
\hline (const & ant) & & 2,36 & & 20,55 & 0,00 \\
\hline \multirow{6}{*}{ 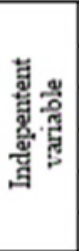 } & RS & & 0,14 & $-0,16$ & $-3,27$ & 0,00 \\
\hline & I & & 0,31 & 0,38 & 7,35 & 0,00 \\
\hline & TD\&E & & $-0,02$ & $-0,03$ & $-0,60$ & 0,55 \\
\hline & WC & & 0,08 & 0,11 & 2,45 & 0,01 \\
\hline & CBPA & & 0,12 & 0,16 & 3,26 & 0,00 \\
\hline & $\mathrm{CR}$ & & 0,06 & 0,08 & 1,89 & 0,06 \\
\hline
\end{tabular}

Dependent variable: employee well-being

The regression analysis showed that EWB is influenced by HRM practices: "RS", "I", "WC" and "CBPA". HRM practices "TD\&E" and "CR" are not statistically significant to EWB. The largest predictor of EWB is HRM practice "I", the second largest predictor "CBPA" and "WC". Furthermore, HRM practice "RS" had a negative influence on EWB.

Equation of regression: $\mathrm{EWB}=2,36-0,14 *(\mathrm{RS})+0,31 *(\mathrm{I})+0,08 *(\mathrm{WC})+0,12 *(\mathrm{CBPA})$

The analyses of mediator effects are performed with statistically significant relationships between HRM practices and EWB obtained from regression analyses. 


\section{Mediation analysis}

To test study hypotheses, mediation analyses were performed.

The mediation analysis has the goal to establish the extent to which a causal variable influences an outcome variable through one or more mediator variables [40]. A simple mediation model contains a predictor variable $(\mathrm{X})$, a mediator variable $(\mathrm{M})$, and an outcome variable (Y) [41] (see Fig. 2: A conceptual diagram of a simple mediation model). Preconditions for mediation analysis are: (1) $\mathrm{X}$ is significantly associated with the $\mathrm{Y}$; (2) $\mathrm{X}$ is significantly associated with the $\mathrm{M}$; and (3) $\mathrm{M}$ is significantly associated with $\mathrm{Y}$ when $\mathrm{X}$ is also included in the regression equation [41]. All cases in this study have met these criteria.

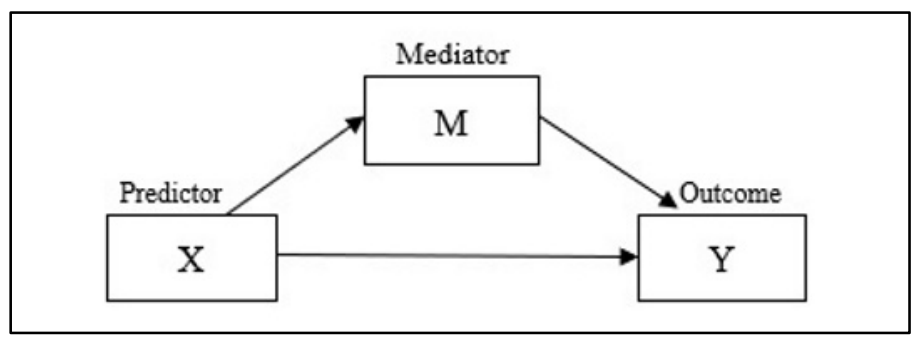

Fig. 2. Conceptual diagram of simple mediation model (source: Hayes, 2018)

According to Baron and Kenny (1986), mediation can be considered full mediation in cases where there is no statistically significant direct effect, but where just an indirect effect is expressed. Meanwhile, mediation can be considered partial mediation in cases where there is both an indirect and a direct statistically significant effect [42].

Summary of the mediation analysis in Table 3, with dependent variable - employee wellbeing, predictor variable - different HRM practices, role of mediator - trust in the organization.

Table 3. Total, direct, and indirect effects of $X$ on $Y$ (source: compiled by author from survey data)

\begin{tabular}{|c|c|c|c|c|c|c|c|c|c|c|c|c|c|c|c|c|}
\hline & \multicolumn{5}{|c|}{ Total effect of $X$ on $Y$} & \multicolumn{5}{|c|}{ Direct effect of $\mathrm{X}$ on $\mathrm{Y}$} & \multicolumn{6}{|c|}{ Indirect effect of $\mathrm{X}$ on $\mathrm{Y}$} \\
\hline & Coef. & se & $t$ & $\mathrm{p}<0,05$ & $\%$ & Coef. & se & $t$ & $\mathrm{p}<0,05$ & $\%$ & Coef. & $\begin{array}{c}\text { Boot } \\
\text { SE }\end{array}$ & $\begin{array}{l}\text { Boot } \\
\text { LLCI }\end{array}$ & $\begin{array}{l}\text { Boot } \\
\text { ULCI }\end{array}$ & $\%$ & $\begin{array}{c}\text { Mediator } \\
\text { effect }\end{array}$ \\
\hline "RS" $\rightarrow$ Trust $\rightarrow$ EWB" & 0,19 & 0,03 & 5,60 & 0,00 & - & $-0,02$ & 0,03 & $-0,48$ & 0,63 & - & 0,20 & 0,03 & 0,15 & 0,25 & - & Full \\
\hline "WC" $\rightarrow$ Trust $\rightarrow$ EWB" & 0,25 & 0,03 & 9,63 & 0,00 & 100 & 0,07 & 0,03 & 2,59 & 0,01 & 29 & 0,18 & 0,02 & 0,14 & 0,22 & 71 & Patrial \\
\hline "CBPA" $\rightarrow$ Irust $\rightarrow$ EWB" & 0,25 & 0,03 & 8,96 & 0,00 & - & 0,05 & 0,03 & 1,86 & 0,06 & . & 0,19 & 0,02 & 0,15 & 0,24 & $=$ & Full \\
\hline
\end{tabular}

"The impact of HRM practice " $X$ " on EWB, mediating trust in the organization.

Bootstrap with 5000 resamples for the analysis $(95 \%$ CI).

Taking into account the obtained results of the mediation analysis, it can be stated HRM practices "RS" and "CBPA" have an indirect effect on EWB through trust in the organization. In these cases, the effect of the mediator's effect is full because the direct effect is not statistically significant $(p>0,05)$. Attention should be paid that the indirect effect is statistically significant in all of the analyzed cases (see Table 3 ) because there is no zero meaning between low (BootLLCI) and high (BootULCI) confidence interval levels.

HRM practices "I" and "WC" have a direct and indirect influence on EWB through the mediator - trust in the organization. In the above-mentioned cases, the mediator's effect is partial.

To compare the calculated percent expressions of the mediator's effect, it can be noted that the indirect effect could explain more than half of the total effect. It shows the indirect mediation effect has a greater influence on EWB than the direct effect. 
Based on the analysis of data, the hypothesis about the trust in the organization effects on relationships between HRM practices: "RS" (1a), "I" (1b), "WC" (1d), "CBPA“ (1e) and EWB are confirmed.

\section{Discussion}

After carrying out a review of current scientific literature, no research was found that the effect of trust in the organization would have a negative effect on the relation between the HRM practices and EWB. It shows this phenomenon is important in the process of the organization's life. By creating a positive, trust-based organizational climate and forming a positive attitude towards the organization, the organization also takes care of its employees and their well-being.

The relationship between trust in the organization and employee well-being is most commonly found in empirical studies in which researchers attempt to examine the impact of leadership style on employee well-being or job satisfaction $[31,35]$. In other cases, perceived organizational policies [32], and socially responsible enterprises [42] have an impact on the well-being of the organization's employees.

A few empirical studies of the analysis of all the three constructs mentioned in the context of this article were found in the scientific literature $[8,30]$. They show the empirical validity of these three constructs is not comprehensive enough. Thus, it would be necessary to keep delving deeper into the effect of trust in the organization on the relation between different HRM practices and EWB in the future.

The results of this study, based on employees in Lithuania, indicate that trust in the organization has a significant mediating effect on the relationship between HRM practices: "RS", "I", "WC", "CBPA" and EWB. The findings of this study contribute to an advanced understanding of HRM practice-EWB relationship by testing the mediation model in the organization context in Lithuania. Thus, it can be stated that the hypotheses (H1a; H1b; H1d; H1e) related with the effect of trust in the organization on these relationships were confirmed.

In contrast to previous studies [44, 17], this research does not confirm a statistically significant relationship between HRM practices "TD\&E", "CR" and EWB. This result may have been affected by the fact that in previous studies the EWB did not consist of life wellbeing, workplace well-being and psychological well-being together. Taking this into account, the effect of trust in the organization on these relationships was not researched in this study. Thus, it can be stated that the hypotheses (H1c; H1f) related with the effect of trust in the organization on these relationships were not confirmed.

To compare the direct effect with the indirect effect in the analyzed relationships, it can be stated that the indirect effect explains over a half of the analyzed cases of the effect in this research. This shows the importance of trust in the organization in order to ensure the employee well-being.

This research shows it is important to ensure interpersonal trust in managers and coworkers as well as in the organization by using the HRM practices in order to achieve the well-being of employees.

\section{Limitations and future direction}

The findings of this study should be interpreted with caution because this study has some limitations. Firstly, the sample size in the present study is fairly small. In generalizing the findings this should be kept in the mind. Secondly, future research may carry out similar longitudinal studies to understand the trend of relationships over an extended period of time. 
Future research may be initiated in other countries and continents to produce reliable and standardized comparisons among different populations.

This study did not research the effect of trust in the organization on the relationships between HRM practice "TD\&E", "CR" and EWB. Further research could be oriented towards the assessment of the indirect effect on these relationships.

This study only considered the mediating effect of trust in the organization between the HRM practices and EWB; therefore, the author suggests that future research could add interpersonal trust as a potential mediator and compare the effects of different mediators. The involvement of different mediators in the analysis would allow seeing a wider view and assess their effect.

It might be fruitful for future studies to investigate the moderating role of trust in the organization on the relationship between HRM practices and employee well-being.

Globalization processes determine the fact that employees of organizations are representatives of different cultures, so the knowledge of cultural differences would help to understand the needs of various organizations' employees more quickly in order to ensure their well-being, especially when companies are becoming more global and are outsourcing their work to areas and cultures different from their own. It is suggested that choosing subgroup representatives of different cultures for future comparative research be taken up in order to assess various cultural influences.

\section{Conclusion}

This study improves our knowledge of connections between HRM practices, trust in the organization, and EWB at the organizational level. Trust in the organization was considered in this study to better understand the process by which HRM practice affects EWB. Furthermore, the results highlight the importance of trust in the organization for employee well-being.

Information about the indirect effect is important because it enables HRM practitioners, and managers, to understand the organization's employees better and to better consider their needs for assurance and support of their well-being. Well-being of employees at work in Lithuania can be improved best by strengthening trust in the organization through following these HRM practices: "RS", "I", "WC" and "CBPA". Much attention is paid to the creation of trust in the organization. Additional research is suggested to explore beyond the identified limitations.

\section{References}

1. D. E. Guest, Hum. Resour. Manag. J., 27(1), 22-38 (2017)

2. M. Beer, P. Boselie, C. Brewster, Hum. Resour. Manag. J., 54(3), 427-438 (2015)

3. R. Peccei, K. Van De Voorde, Hum. Resour. Manag. J., 29(4), 539-563 (2019)

4. S. Hauff, M. Guerci, S. Gilardi, Evidence-based HRM, 8(3), 253-271 (2020)

5. B. Cooper, J. Wang, T. Bartram, F. L. Cooke, Hum. Resour. Manag. J., 58(1), 85-97 (2019)

6. M. J. Ashleigh, M. Higgs, V. Dulewicz, Hum. Resour. Manag. J., 22(4), 360-376 (2012)

7. M. Vanhala, R. Ahteela, Manag. Res. Rev., 34(8), 869-888 (2011)

8. K. Alfes, A. Shantz, C. Truss, Hum. Resour. Manag. J., 22(4), 409-427 (2012)

9. A. Richter, K. Näswall, Work \& Stress, 33(1), 22-40 (2019) 
10. D. Fan, L. Cui, M. M. Zhang, C. J. Zhu, C. E. Härtel, C. Nyland, Int. J. Hum. Resour. Manag., 25(7), 931-950 (2014)

11. W.-R. Huang, C.-H. Su, Ind. Commer. Train., 48(1), 42-52 (2016)

12. X. Zhang, Z. Lin, Y. Liu, X. Chen, D. M. Liu, Empl. Relat.: Int. J., 42(4), 903-919 (2020)

13. P. Agarwal, E. Farndale, Hum. Resour. Manag. J., 27(3), 440-458 (2017)

14. D. T. Kooij, D. E. Guest, M. Clinton, T. Knight, P. G. Jansen, J. S. Dikkers, Hum. Resour. Manag. J., 23(1), 18-35 (2013)

15. J. Korff, T. Biemann, S. C. Voelpel, J. Organ. Behav., 38(1), 45-67 (2017)

16. K. Van De Voorde, J. Paauwe, M. Van Veldhoven, Int. J. Manag. Rev., 14(4), 391-407 (2012)

17. G. Demo, E. R. Neiva, I. Nunes, K. Rozzett, BAR-Brazilian Administration Review, 9(4), 395-420 (2012)

18. A. Salas-Vallina, M. Pozo, R. Fernandez-Guerrero, Empl. Relat.: Int. J., 42(3), 561-581 (2020)

19. N. Dimotakis, B. A. Scott, J. Koopman, J. Organ. Behav., 32(4), 572-588 (2011)

20. C. L. M. Keyes, Soc. Psychol. Quart., 61(2), 121-140 (1998)

21. E. A. Locke, D. P. Latham, Psychol. Sci., 1(4), 240-246 (1990)

22. C. D. Fisher, Int. J. Manag. Rev., 12, 384-412 (2010)

23. K. M. Page, D. A. Vella-Brodrick, Social indicators research, 90(3), 441-458 (2009)

24. X. Zheng, W. Zhu, H. Zhao, C. Zhang, J. Organ. Behav., 36(5), 621-644 (2015)

25. D. M. Rousseau, S. B. Sitkin, R. S. Burt, C. Camerer, Acad. Manag. Rev. (AMR), 23(3), 393-404 (1998)

26. R. C. Mayer, J. H. Davis, J. Appl. Psychol., 84(1), 123 (1999)

27. S. H. Haynes, M. C. Leone, L. D. Keena, D. C. May, R. Ricciardelli, E. G. Lambert, J. Crim. Justice, 43(5), 623-639 (2020)

28. M. Vanhala, K. Puumalainen, K. Blomqvist, Person. Rev., 40(4), 485-513 (2011)

29. E. Güçer, A. D. Şerif, Bus. Man. Dyn. (BMD), 4(1), 12 (2014)

30. A. F. Oliveira, Gomide Jr., B. V. Poli, Revista de Administração Mackenzie, 21(1) (2020)

31. S. Braun, C. Peus, S. Weisweiler, D. Frey, The Leadership Quarterly, 24(1), 270-283 (2013)

32. S. Ullah, S. A. Hasnain, A. Khalid, A. Aslam, European online journal of natural and social sciences 8(1), 1-14 (2019)

33. R. Bachmann, N. Gillespie, R. Priem, Organ. Stud., 36(9), 1123-1142 (2015)

34. J. Paliszkiewicz, The importance of building and rebuilding trust in organizations, In Diversity, Technology, and Innovation for Operational Competitiveness: Proceedings of the 2013 International Conference on Technology Innovation and Industrial Management, 269-278 (2013)

35. T. W. Ng, J. Vocat. Behav., 88, 154-163 (2015)

36. S. Y. Chen, W. C. Wu, C. S. Chang, C. T. Lin, J. Y. Kung, H. C. Weng, ... S. I. Lee, BMC health services research, 15(1), 1-17 (2015)

37. P. Mehta, Benchmarking: An International Journal (2020) 
38. M. Vanhala, VINE Journal of Information and Knowledge Management Systems, 50(2), 349-368 (2019)

39. P. A. Kennedy, Guide to econometrics, (John Wiley \& Sons, 2008)

40. A. F. Hayes, Introduction to mediation, moderation, and conditional process analysis (2018)

41. P. E. Jose, Doing Statistical Mediation and Moderation. (D. A. Kenny \& T. D. Little, Eds, 2013)

42. R. M. Baron, D. A. Kenny, J. Pers. Soc. Psychol., 51(6), 1173. (1986)

43. Y. Yu, Y. Choi, Chinese Management Studies, 8(4), 577-592. (2014)

44. G. Demo, T. Paschoal, Paidéia (Ribeirão Preto), 26(63) (2016) 